\title{
Introduction to the Special Issue on Cultural Adaptations of CBT
}

Cognitive behaviour therapy (CBT) has become the first line of treatment for a wide range of mental health problems across many countries. The flexibility of CBT, and a strong evidence base and good track record of training and dissemination, has lent itself to scaling up of this therapy, and projects such as the Improving Access to Psychological Therapies (IAPT) programme in England have shown that large-scale projects to roll out CBT at a national level are possible (Clark, 2011).

CBT was a therapy initially developed, refined and disseminated by therapists, trainers and researchers from ethnic, religious and sexual majority backgrounds (i.e. White, agnostic/Christian and heterosexual - although we acknowledge these cultural issues were rarely reflected on, as often happens with majority identity factors). The subsequent ubiquity of CBT has raised the challenge of demonstrating that it is a model of therapy that can be adapted at an international level for diverse populations in Western contexts and in low- and middle-income countries, where there are challenges both in adapting the content to local values and practices, and working with considerably fewer resources than in Western contexts (e.g. Bennett-Levy et al., 2014; Naeem et al., 2014, 2015; Beck, 2016).

Therapists and researchers have begun to rise to this challenge over the past 20 years. Initially in the UK this work began to happen in HIV services as therapists from ethnic and sexual minority backgrounds began to adapt therapies for men who have sex with men, and largely heterosexual sub-Saharan African service users (Tallis, 1995). Soon these initiatives developed momentum as minority therapists in other areas began to adapt therapies for diverse populations and majority therapists began to recognize the limits of models of therapy that did not take into account the culture and context of service users. This adaptation was further developed by therapists focusing on trauma-focused CBT (TF-CBT), who began to specialize in work with asylum seekers and refugees, and with therapists working in psychosis services, where Black and Minority Ethnic service users have historically worse outcomes. A growing awareness of institutionalized racism and discrimination also empowered therapists to look critically at the degree to which these processes were evident in therapy provision (Beck, 2005).

Cultural adaptations need to take into account the lived experience of patients and the particular values, beliefs about health, lifestyles and spirituality that they may have, as well as acknowledging that being in a minority group can be a risk factor for mental health problems due to the marginalization, poverty and discrimination that people are much more likely to experience if they are members of these groups (e.g. transgender individuals; Austin and Craig, 2015). Balancing these adaptations whilst keeping true to the core, evidence-based principles of CBT can be a challenge and we salute those clinicians, researchers and writers who have embarked on this endeavour. Within this special issue we have welcomed contributions looking at both adaptations for specific cultures 
within a Western context but also cultural adaptations of CBT for individuals in nonWestern countries for whom adaptations may be required to take into account specific factors.

In embracing a broad definition of 'culture' (to mean customs and beliefs of a group of people at a particular time), this special issue could not hope to cover every possible adaptation required. For example, we hoped for, but did not receive, any papers on adapting CBT for individuals from low socioeconomic backgrounds, from South America or with people who are members of gangs or who have particular sub-cultural affiliations. However, we hope that it gives therapists a flavour of what kind of adaptations can be creatively made within an evidence-based framework, and some principles that can be reflectively generalized to other groups and communities.

We would argue that cultural competence is required to be a competent CBT therapist. Whilst one cannot expect all CBT therapists to be experts in all cultures, it is reasonable to expect the competent CBT therapist to reflect on their own culture, beliefs and assumptions to ensure that these are not interfering with the delivery of therapy. More than this, it is wise to be curious and willing to learn about other cultures and adapt therapy content and delivery methods to maximize this for the various differing cultures that therapists might encounter in their day-to-day work.

Currently, many health professional associations already explicitly refer to cultural competence within their code of conduct (Haarhoff and Thwaites, 2016), although as yet the British Association for Behavioural and Cognitive Psychotherapies (BABCP) does not include this within their Standards of Conduct, Performance and Ethics (BABCP, 2018), limiting their reference to this area to:

You must not allow your views about a service user's sex, age, colour, race, disability, sexuality, social or economic status, lifestyle, culture, religion or beliefs to affect the way you treat them or the advice you give. You must treat service users with respect and dignity. If you are providing care, you must work in partnership with your service users and involve them in their care as appropriate (p. 6).

It is a real pleasure, then, to look at the sheer breadth of work presented in this special issue on culturally adapted CBT. Authors are a mixture of some of the pioneers in this field with decades of research and clinical experience underpinning their expertise, alongside authors who are publishing for the first time and just starting their academic careers. Many submitted outstanding papers but were worried about the content not being good enough or being unsupported by their employers in terms of being given time and resources to disseminate their excellent work. The editorial team have particularly enjoyed being part of their journey to publication and look forward to seeing this next generation of clinician-researchers continuing to expand our understanding of how CBT needs to adapt to take into account an increasingly diverse world. We are in discussions with the BABCP Equality and Culture Special Interest Group regarding how the wider CBT research community can support frontline clinicians to produce research that captures the challenges and potential creative answers in this area.

One important point is that whilst this special issue aims to provide a dedicated focus on the cultural adaptations of CBT, this is not the end-point; nor does the Cognitive Behaviour Therapist consider that such articles would not fit within usual future issues. We would 
welcome further submissions on this topic for future issues - cultural adaptions should be considered part of the mainstream of CBT.

LYDIA STONE, ANDREW BECK, AND FARAMARZ HASHEMPOUR

(Guest Editors, tCBT)

RICHARD THWAITES

(Editor-in-Chief, tCBT)

\section{References}

Austin A, Craig SL (2015). Transgender affirmative cognitive behavioral therapy: Clinical considerations and applications. Professional Psychology: Research and Practice 46, 21-29. https://doi.org/10.1037/a0038642

Beck A (2005). Identifying institutionalised racism in psychology services: a prospective, quantitative study of ethnic minority service use and treatment outcomes in a sexual health / HIV service. Clinical Psychology 49, 36-40.

Beck A (2016). Transcultural Cognitive Behavioural Therapy for Anxiety and Depression: A Practical Guide. Abingdon, UK: Routledge.

Bennett-Levy J, Wilson S, Nelson J, Stirling J, Ryan K, Rotumah D et al. (2014). Can CBT be effective for Aboriginal Australians? Perspectives of Aboriginal practitioners trained in CBT. Australian Psychologist 49, 1-7. doi: 10.1111/ap.12025

British Association for Behavioural and Cognitive Psychotherapies (BABCP) (2017). Standards of Conduct, Performance and Ethics. Available at: http://www.babcp.com/About/Documents.aspx

Clark DM (2011). Implementing NICE guidelines for the psychological treatment of depression and anxiety disorders: the IAPT experience. International Review of Psychiatry 23, 375-384.

Haarhoff B, Thwaites R (2016). Reflecting on our socio-cultural background: becoming more culturally sensitive and effective CBT therapists. In Reflection in CBT, Haarhoff BA, Thwaites R (eds), pp. 77-93. London: Sage. doi: 10.4135/9781473919952.n6

Naeem F, Saeed S, Irfan M, Kiran T, Mehmood N, Gul M et al. (2015). Brief culturally adapted CBT for psychosis (CaCBTp): a randomized controlled trial from a low-income country. Schizophrenia Research 164, 143-148. doi: 10.1016/j.schres.2015.02.015

Naeem F, Sarhandi I, Gul M, Khalid M, Aslam M, Anbrin A, Saeed S et al. (2014). A multicentre randomised controlled trial of a carer supervised culturally adapted CBT (CaCBT) based self-help for depression in Pakistan. Journal of Affective Disorders 156, 224-227. https://doi.org/10.1016/j.jad.2013.10

Tallis F (1995). Cognitive behavioural strategies for HIV sexual risk reduction. Clinical Psychology and Psychotherapy 2, 267-277. 